\title{
Backpropagation Neural Network Algorithm for Water Level Prediction
}

\author{
Subianto \\ Magister of Information System \\ Diponegoro University
}

\author{
Suryono \\ Magister of Information System \\ Diponegoro University
}

\author{
Jatmiko Endro Suseno \\ Magister of Information System \\ Diponegoro University
}

\begin{abstract}
Floods cause economic losses, even cause loss of life. To anticipate floods and the impacts, flood prediction including early warning systems should be developed using appropriate techniques. The aim of this research is to apply the back propagation neural network algorithm for water level prediction and produce web-based flood prediction information system. The system is built using a back propagation neural network algorithm. This algorithm has 3 stages in the training process, which are forward feed, calculation, and back propagation. The used data is derived from the physics laboratory of Diponegoro University. This study concludes that the application of back propagation neural network algorithm for flood prediction can produce an accurate prediction. Therefore, this can be a reference for predicting floods significantly based on water levels in certain places. In this study obtained MSE at the first iteration of 0.0142 , the smallest MSE that meets the limit of threshold of 0.000002420 and data accuracy of $98.66 \%$. This means that generally, the back propagation neural networks application produce accurate water level prediction, which is close to the actual data.
\end{abstract}

\section{General Terms}

Water Level Prediction

\section{Keywords}

Artificial neural network, backpropagation, prediction, water level, flood, early warning.

\section{INTRODUCTION}

Floods cause economic losses or even cause loss of life. This overflowing / flooding occurs because of the unaccommodating water flowing in the right or in the left side of the river area / waterways due to the river does not have sufficient capacity for the passing flow. Flooding occurs when the volume of water flow increase relatively large, the water flows out of the river and is said to be flooded if water flows out of the river channel and causes disturbance to humans [4]. Flooding is also caused by processes related to sea level and water flow [9]. Rising water levels in rivers caused severe flooding and puddles of water, prompting research to monitor flooding on the Huahei River [14].

Flooding is a common natural disaster and causes serious and painful damage. The estimated loss caused by the flood compared to all other disasters is about $40 \%$. Floods occur frequently, affecting large areas [5]. To anticipate floods and impacts, appropriate techniques should be developed for flood prediction [10].

Predictions of flooding are difficult because of weather anomalies [14]. Therefore, back propagation neural networks are used in applications for flood forecasting. This algorithm allows the use of several variables both in input and output layers. This method has been shown to provide better results in performance and efficiency [3].

The technology of back propagation neural networks can be used to complement the processing of information from network through the interaction of nerve cells. Mapping of stimulant effects and output input estimations are obtained through a combination of nonlinear functions. This provides the advantage of self-learning, self-organizing, self-adaptation and fault tolerance. This method is feasible to use for flood prediction [3].

The water level criteria data on the upstream and downstream sections contain a pattern that can be used as data for prediction and generate EWS (Early Warning System) [7]. EWS is a tool consisting of a series of mechanisms and procedures for detecting hazards, monitoring indicators, warning communications and alarms and evacuation of vulnerable populations to safe areas [8].

Wireless Sensor Network (WSN) became one of the tools that able to communicate with the computer without going through the media cable. WSN ultrasonic sensor systems have been developed for water level measurements by utilizing telemetry data from water level sensors multi point and real time using TCP / IP communication protocol. The availability of fluctuating data that can be recorded in real time becomes an obstacle in measuring water levels in flood prediction. Real time data obtained with WSN will be utilized in constructing high water prediction information system at certain point with back propagation neural network algorithm, so it is expected to produce real time information quickly, precisely and accurately [12].

From the background mentioned above then conducted research on the implementation of artificial neural network algorithm back propagation to predict water level.

\section{ARTIFICIAL NEURAL NETWORK}

Artificial neural networks are parallel processor systems connected to each other in graphical form directed according to the chart of each neuron of the network represented as a node. This connection provides a hierarchical structure that tries to mimic brain physiology, searching for new processing models to solve specific problems in the real world. An important problem in the development of artificial neural network techniques is useful behavior that must be learned, acknowledged, and applied between objects and real-world object frames [2].

Artificial neural network consists of 3 layers, namely Input Layer, Hidden Layer and Output Layer, as shown in figure 1. Each layer is responsible for performing the same functionality as completing the system. This structure is based 
on modifications of the three-layer model of software architecture, these are: Data layer, service layer (web, sensor) and presentation layer (web, windows and agent) [13].

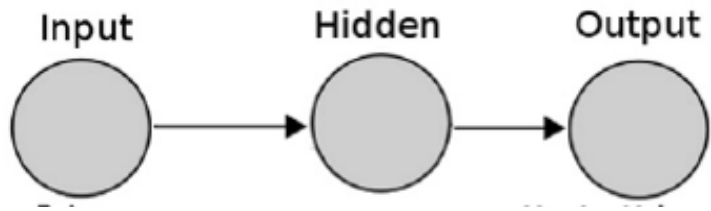

Fig 1: Neural Networks basic schema

Artificial neural network is determined by 3 important things: Pattern relationship between neurons, methods to determine the weight (training learning) and activation functions.

\section{BACK PROPAGATION NETWORK}

One model of Artificial Neural Networks used to solve problems is the Back Propagation Network [11]. This model is used because in most cases it can recognize the required pattern, identifying when the facts or phenomena will happen again before it happens.

The back propagation network architecture has a hidden layer, although it can basically have more than one hidden layer according to system requirements. While the number of vertices connected to the input layer and the output layer is determined by the number of input patterns and the number of output patterns.

Training on a neural network is training to determine the appropriate weighting value. Training on the back propagation network consists of three stages: advanced feed training, error calculation (calculation and error reshuffling) and weight adjustment. The architecture and processes in the back propagation network algorithm are shown in figure 2 .

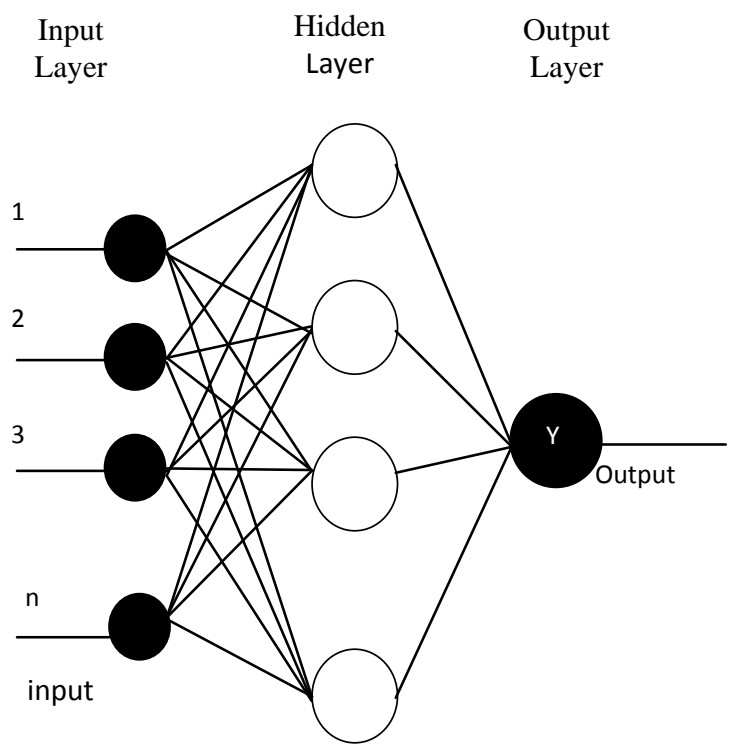

Fig 2: BPN Architecture

The Activation function is a mathematical operation imposed on the output signal $\mathrm{y}$. This function is used to enable or disable neurons. The behavior of the ANN is determined by the specified weight and activation function. The activation function used is sigmoid binner.

This activation function is used in ANN using backpropagation. This function has a range of 0 to 1 then its outpuy value lies at interval 0 to 1 or its output value is 0 or 1 .
The formula of the binary sigmoid activation function is shown in figure 3 . In matlab the binary sigmoid activation function is known as logsig [6].

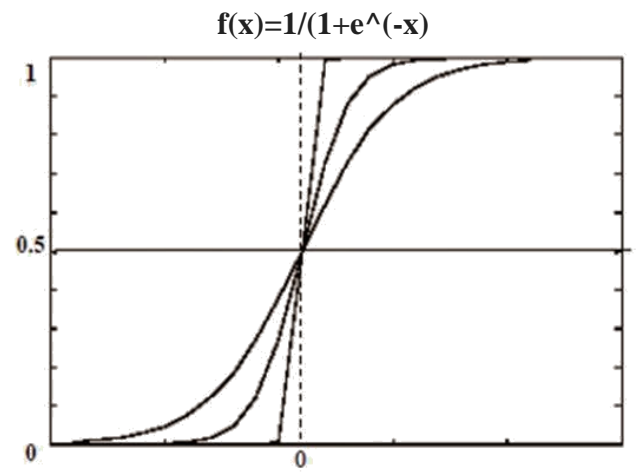

Fig 3: Sigmoid binner

The propagation algorithm is described in the following steps or procedure flow:

Artificial Neural Network Training Process Back Propagation:

Step 0: Initialize all weights with small random numbers, epoch $=1$, specify the learning rate $(\alpha)$, momentum $(\beta)$, specify the number of units on the hidden layer (p) and specify the termination condition. The termination conditions are max epoch and target error.

Step 1: If epoch $\neq$ max epoch and target error $<\mathrm{MSE}$, do step 2-9.

Step 2: For each pair of training data (1 to a where a is the amount of training data), do steps 3-8.

\section{Phase I: Feed Forward}

Step 3: Each input unit receives the signal and passes it to the hidden unit above it.

Step 4: Calculate all outputs in hidden units zj $(j=1,2, \ldots, p)$

$$
z_{-} n e t_{j}=v_{j 0}+\sum_{i=1}^{n} x_{i} v_{j i}
$$

If we use the sigmoid activation function, calculate the output by:

$$
z_{j}=f\left(z_{-} n e t_{j}\right)=\frac{1}{1+e^{-z_{-} n e t_{j}}}
$$

Step 5: Calculate all network outputs in unit yk $(\mathrm{k}=1,2, \ldots$, $\mathrm{m})$

$$
y_{-} \text {net }_{k}=w_{k 0}+\sum_{j=1}^{p} z_{j} w_{k j}
$$

If we use the sigmoid activation function, calculate the output by:

$$
y_{k}=f\left(y_{-} n e t_{k}\right)=\frac{1}{1+e^{-y_{-} n e t_{k}}}
$$

Phase II: Back propagation

Step 6: Calculate $\delta$ output unit based on error in each output unit $\mathrm{yk}(\mathrm{k}=1,2, \ldots, \mathrm{m})$

Calculate the rate of $\mathrm{Wkj}$ weight change with $\alpha$ acceleration rate (momentum). 


$$
\Delta w_{k j}=\alpha \delta_{k} z_{j}
$$

$k=1,2, \ldots, m ; j=0,1, \ldots, p$

Step 7: Calculate $\delta$ hidden unit based on error in each hidden unit $\mathrm{z} \mathrm{j}(\mathrm{j}=1,2, \ldots, \mathrm{p})$

$$
\delta_{-} n e t_{j}=\sum_{k=1}^{m} \delta_{k} w_{k j}
$$

$$
\delta j=\delta_{-} n e t_{j} f^{\prime}\left(z_{-} n e t_{j}\right)=\delta_{-} n e t_{j} z_{j}\left(1-z_{j}\right)
$$

Calculate the vji weight change with $\alpha$ acceleration rate

$$
\Delta v_{j i}=\alpha \delta_{j} x_{i}
$$

$\mathrm{j}=1,2, \ldots, \mathrm{p} ; \mathrm{i}=0,1, \ldots, \mathrm{n}$

\section{Phase III: Change of weight}

Step 8: Calculate all weight changes.

The weight change of the line leading to the output unit:

$$
w_{k j}(n e w)=w_{k j}(n o w)+\Delta w_{k j}
$$

$\mathrm{k}=1,2, \ldots, \mathrm{m} ; \mathrm{j}=0,1, \ldots, \mathrm{p}$

Weight changes leading to hidden units:

$$
v_{k j}(n e w)=v_{k j}(\text { now })+\Delta v_{j i}
$$

$j=1,2, \ldots, p ; i=0,1, \ldots, n$

Step 9: Update epoch value.

$$
\text { epoch }=\text { epoch }+1
$$

Then, the network output is compared to the target, calculate the E error using:

$$
E=t-y_{k}
$$

To calculate the mean squared error (MSE), it is formulated:

$$
M S E=\frac{\sum_{k=1}^{a}\left(t_{k}-y_{k}\right)^{2}}{a}
$$

The number of input nodes adjusted to the amount of data to be fed on the network. In this design, the data of water level pattern of 5 minute data interval is used. Data entered 5 minutes before for each process. The resulting output is the predicted water level 5 minutes forward as shown in Figure 4. The process of reading patterns into information is used to determine the rules that will be used as a selection process of input data and used to determine the minimum selection process of the rules of a flood early warning system.
Input

Output

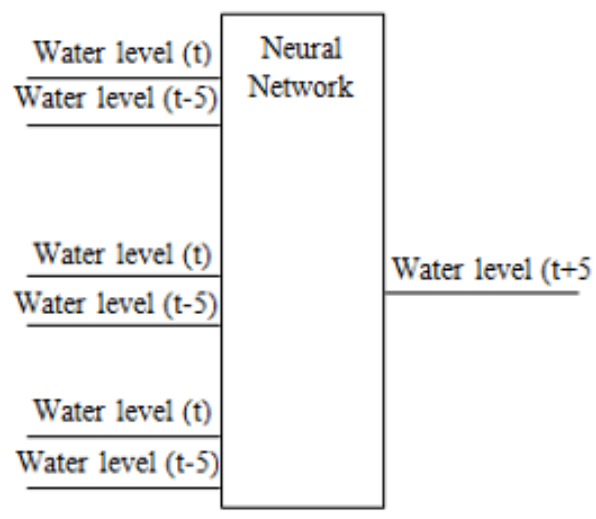

Fig 4: System Process

The calculation of this water level starts from the downstream to the upstream by setting a certain point as the starting point of the calculation.

The status of water elevation was determined by the Directorate General of Water Resources, Ministry of Public of Works and Housing through Circular Letter No. 5 / SE / D / 2016. The level of danger will be conveyed to not use the term confusing or generating multi-interpretation. The highest level of danger to use the red alert term, the danger level is using the yellow standby term, and the degree of light danger of using the green standby term, with the following criteria [1]:

a. Red alert: height of care $<0.8 \mathrm{~m}$

b. Yellow alert: care height $0.8 \mathrm{~m}-1.20 \mathrm{~m}$

c. Green alert: height of care $>1,20 \mathrm{~m}$

d. Blue alert: height of care $>1.50 \mathrm{~m}$

\section{DESIGN OF RESEARCH}

\subsection{Materials and Tools}

Materials and tools used to conduct research on the implementation of artificial neural network back propagation network algorithm for the prediction of water level is the actual data which is the result of observation using wireless sensor network at laboratory Instrumentation and Electronics Department of Physics which is measured and recorded by laboratory Instrumentation and Electronics Department of Physics Diponegoro University of Semarang. Actual data used consist of time elements with a certain time interval and water level data. The tool used in this research are PHP and MySQL.

\subsection{Research Procedure}

The research procedure of the implementation back propagation neural network algorithm for prediction of water level is shown in figure 5 . 


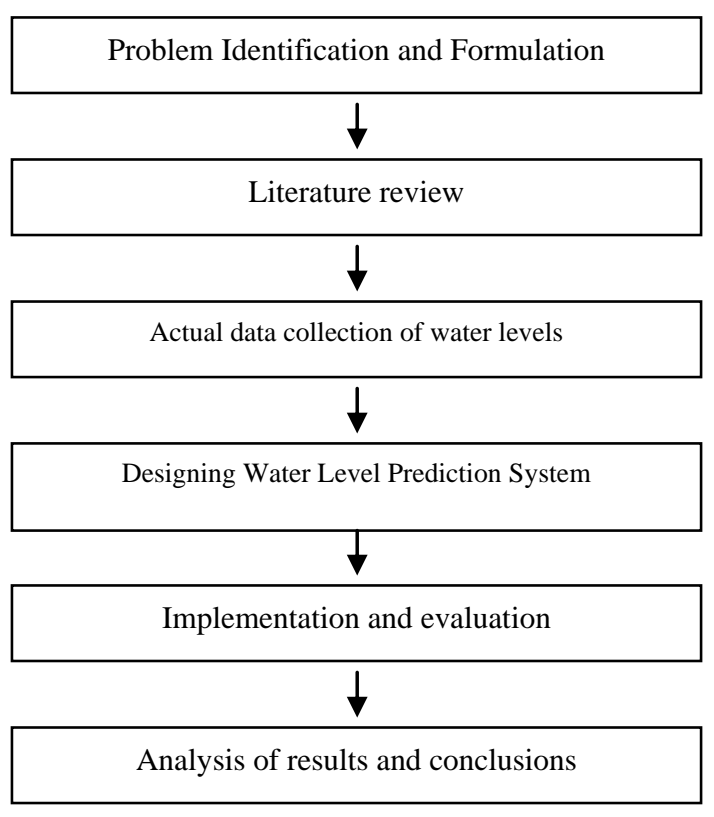

Fig 5: Research procedure

\subsection{Flowchart Information System}

The system design stage that describes 9 sequences of back propagation process in the prediction water level information system can be described in the symbols of the flow chart in detail in figure 6.

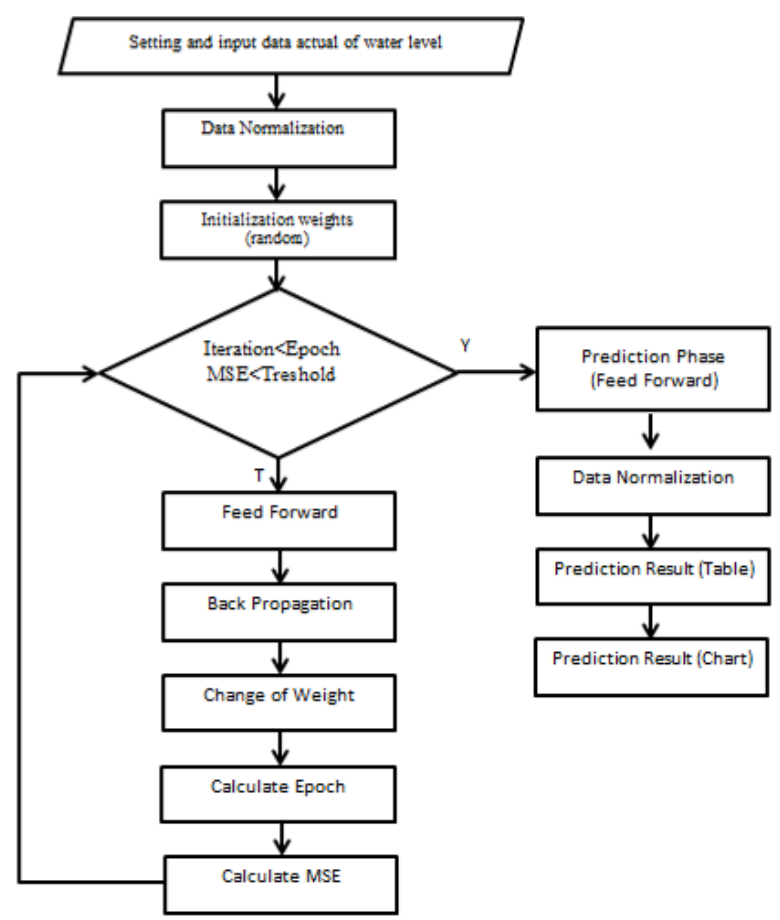

Fig 6: Flowchart of Information System

\subsection{Entity Relationship Diagram}

The design of entity relationship diagram for the information system of the application of back propagation neural network algorithm for prediction of water level is shown in Figure 7.

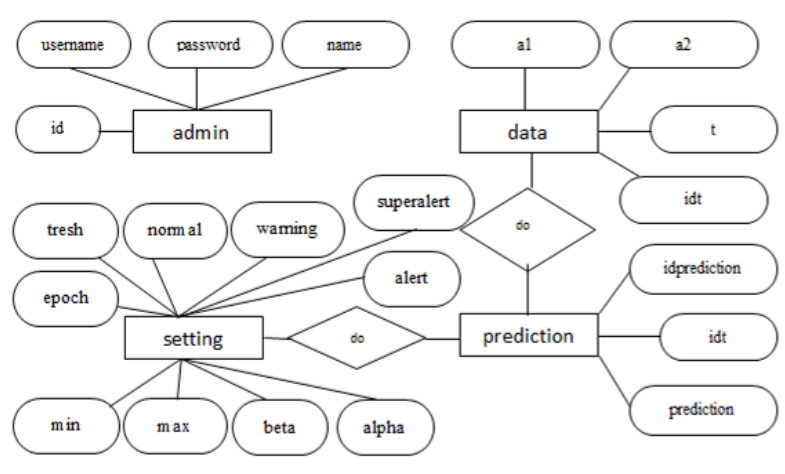

Fig 7: ER Diagram

\subsection{Physical Data Model}

\subsubsection{User Login Table}

User login table used to store users who can login, as shown in Table 1.

Table 1: Login User

\begin{tabular}{|c|c|c|c|}
\hline Attribute & Type & Length & Detail \\
\hline id & integer & 2 & Primary Key(ai) \\
\hline username & varchar & 15 & User \\
\hline password & varchar & 15 & Word key / code \\
\hline name & varchar & 20 & Name of user \\
\hline
\end{tabular}

\subsubsection{Data Table}

The data table is used to store the actual data consisting of idt, $\mathrm{t}$ (time), a1 (real data) and a2 (target data), as shown in table 2.

Table 2: Data Table

\begin{tabular}{|c|c|c|c|}
\hline Attribute & Type & Length & Detail \\
\hline idt & integer & 5 & Primary Key(ai) \\
\hline T & time & 10 & Time \\
\hline a1 & varchar & 10 & Water level t-5 \\
\hline a2 & varchar & 20 & Water level t \\
\hline
\end{tabular}

\subsubsection{Setting Table}

The settings table is used to store settings data, consisting of idsetting, tresh (treshold), alpha, beta, epoch, minimal, maximal, normal, warning and alert. The design of the setting table is shown in table 3.

Table 3: Setting Table

\begin{tabular}{|c|c|c|c|}
\hline Attribute & Type & Length & Detail \\
\hline idsetting & int & 11 & Primary Key(ai) \\
\hline tresh & float $(11,5)$ & 11,5 & treshold \\
\hline alpha & float(1,1) & 1,1 & Alpha \\
\hline beta & float $(1,1)$ & 1,1 & Beta \\
\hline epoch & $\operatorname{int}(11)$ & 11 & Epoch \\
\hline
\end{tabular}




\begin{tabular}{|c|c|c|c|}
\hline minimal & float $(11,5)$ & 11,5 & maxnumber \\
\hline maximal & float(11,5) & 11,5 & minnumber \\
\hline Normal & float(11,5) & 11,5 & Water level warning \\
\hline warning & float(11,5) & 11,5 & Water level warning \\
\hline Alert & float(11,5) & 11,5 & Water level warning \\
\hline superalert & float $(11,5)$ & 11,5 & Water level warning \\
\hline
\end{tabular}

\subsubsection{Prediction Table}

The prediction table is used to store predictive data, which consists of idprediction, idt and prediction. The predictive table design is shown in Table 4.

Table 4: Prediction Table

\begin{tabular}{|c|c|c|c|}
\hline Attribute & Type & Length & Detail \\
\hline idprediction & Int & 11 & Primary Key(ai) \\
\hline Idt & Int & 5 & Id data \\
\hline prediction & Float & 5,5 & Prediction (result) \\
\hline
\end{tabular}

\section{RESULTS}

\subsection{Information System Framework}

The design of water level prediction information system by applying back propagation neural network algorithm begins with the actual data entry stage of time and water level which is then stored in the database. The next stage is the water level prediction process for the next 5 minutes. The prediction process begins with the training phase. In the process of recording data / data entry, data at time $t+5$ will be targeted in the training process. Predicted results are displayed in the form of data tables and graphs. This is shown in the system framework shown in figure 8 .

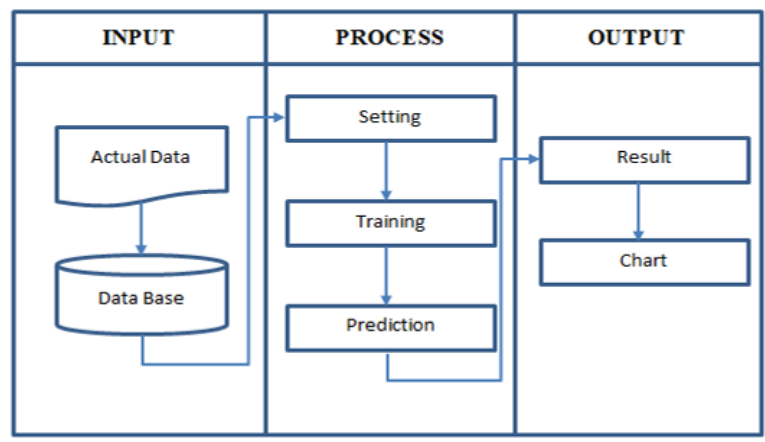

Fig 8: Framework

The data for the training process are: input layer $=1$, using data $t$ (real data based on data retrieval time). The target data used is $t+5$ (data derived from data retrieval in the time range 5 minutes later from the time of data retrieval). These data are presented in table 5 .

Table 5: Data

\begin{tabular}{|c|c|c|}
\hline TIME (t) & DATA & TARGET $(\mathbf{t}+\mathbf{5})$ \\
\hline $12: 00$ & 96 & 94.8 \\
\hline $12: 05$ & 94.8 & 90.9 \\
\hline $12: 10$ & 90.9 & 92 \\
\hline $12: 15$ & 92 & 94.7 \\
\hline
\end{tabular}

\begin{tabular}{|c|c|c|}
\hline $12: 20$ & 94.7 & 95.7 \\
\hline $12: 25$ & 95.7 & 98 \\
\hline $12: 30$ & 98 & 99.9 \\
\hline $12: 35$ & 99.9 & 102.1 \\
\hline $12: 40$ & 102.1 & 103 \\
\hline $12: 45$ & 103 & 101.4 \\
\hline $12: 50$ & 101.4 & 100.2 \\
\hline $12: 55$ & 100.2 & 97.7 \\
\hline $13: 00$ & 97.7 & 97 \\
\hline $13: 05$ & 97 & 96.3 \\
\hline $13: 10$ & 96.3 & 97 \\
\hline $13: 15$ & 97 & 96.8 \\
\hline $13: 20$ & 96.8 & 96.5 \\
\hline $13: 25$ & 96.5 & 97 \\
\hline $13: 30$ & 97 & 97.5 \\
\hline $13: 35$ & 97.5 & 97.9 \\
\hline $13: 40$ & 97.9 & 98 \\
\hline $13: 45$ & 98 & 98.6 \\
\hline $13: 50$ & 98.6 & 99 \\
\hline $13: 55$ & 99 & 100.1 \\
\hline $14: 00$ & 100.1 & 100.3 \\
\hline $14: 05$ & 100.3 & 100.7 \\
\hline $14: 10$ & 100.7 & 101.1 \\
\hline $14: 15$ & 101.1 & 101.4 \\
\hline $14: 20$ & 101.4 & 102 \\
\hline $14: 25$ & 102 & 102.3 \\
\hline $14: 30$ & 102.3 & 102.9 \\
\hline $14: 35$ & 102.9 & 103.1 \\
\hline $14: 40$ & 103.1 & 103 \\
\hline $14: 45$ & 103 & 103 \\
\hline $14: 50$ & 103 & 102.8 \\
\hline
\end{tabular}

The next process is normalization (process scale) to the data in the prediction system. The normalization process is performed so that the output of this back propagation neural network matches the activation function used. The interval for the normalization process of these data is $[0,1]$, this is because the prediction of the water level can be either positive or 0 .

Normalization of data should be done at smaller intervals $(0.1$ - 0.9). This is done because the sigmoid function never reaches a value of 0 or 1 . The formula used in data normalization is:

$$
t=(H I-L O)\left(\frac{x-\operatorname{Min}}{M a x-M i n}\right)+L O
$$

$\mathrm{t}$ is the result of normalization, $\mathrm{HI}$ is the upper limit of the interval (eg 0.9), LO is the lower limit of the interval (eg 0.1), $\mathrm{x}$ is the data to be transformed, Min is the smallest data of the data set and Max is the largest data in the data set. 
After the data is normalized, it will be tested by using generate input weight to the initial hidden, input bias to hidden, hidden weight to output, and hidden bias to output. Weight data is generated randomly.

The settings used in the water level prediction information system by applying this back propagation neural network algorithm are as follows: alpha value (momentum) 0.3 , beta value (learning rate) 0.1, maximum value of data 103.1, minimum value of data 90.9. Number of layer 3 , hidden layer 8 and output layer 1 , treshold value 0.0001 , maximum epoch 10000 .

In the training process, the number of iterations (epoch) are 2880. The limit of the treshold value is achieved at the iteration to 2880 , the MSE value 0.00009984 is smaller than the treshold limit.

The test phase produces a data output that is still within the 0.1 - 0.9 interval. The output data generated must be converted back (normalized) to the original data form by the formula:

$$
t=(\operatorname{Max}-\operatorname{Min})\left(\frac{x-L O}{H I-L O}\right)+\operatorname{Min}
$$

$\mathrm{t}$ is the result of normalization, $\mathrm{HI}$ is the upper limit of the interval (eg 0.9), LO is the lower limit of the interval (eg 0.1), $\mathrm{x}$ is the data to be transformed, Min is the smallest data of the data set (90.9) and Max is the data largest in the data set (103.1).

The next step is to calculate the level of prediction accuracy. This is done by comparing the predicted result $t+5$ with the target data t. The results show that the average accuracy of the average prediction is $99.97 \%$. This indicates that the water level prediction information system by applying a back propagation neural network algorithm can recognize patterns of water level data. The predicted result of water level is shown in table 6.

Table 6: Result

\begin{tabular}{|r|r|r|c|}
\hline Time & \multicolumn{1}{|c|}{ Data } & \multicolumn{1}{c|}{ Prediction } & Status \\
\hline $12: 00$ & 96 & 94.8 & Yellow Alert \\
\hline $12: 05$ & 94.8 & 91 & Yellow Alert \\
\hline $12: 10$ & 90.9 & 91.9 & Yellow Alert \\
\hline $12: 15$ & 92 & 94.5 & Yellow Alert \\
\hline $12: 20$ & 94.7 & 95.6 & Yellow Alert \\
\hline $12: 25$ & 95.7 & 97.9 & Yellow Alert \\
\hline $12: 30$ & 98 & 99.8 & Yellow Alert \\
\hline $12: 35$ & 99.9 & 102 & Yellow Alert \\
\hline $12: 40$ & 102.1 & 102.9 & Yellow Alert \\
\hline $12: 45$ & 103 & 101.4 & Yellow Alert \\
\hline $12: 50$ & 101.4 & 100.2 & Yellow Alert \\
\hline $12: 55$ & 100.2 & 97.7 & Yellow Alert \\
\hline $13: 00$ & 97.7 & 97 & Yellow Alert \\
\hline $13: 05$ & 97 & 96.3 & Yellow Alert \\
\hline $13: 10$ & 96.3 & 97 & Yellow Alert \\
\hline $13: 15$ & 97 & 96.8 & Yellow Alert \\
\hline $13: 20$ & 96.8 & 96.5 & Yellow Alert \\
\hline
\end{tabular}

\begin{tabular}{|r|r|r|c|}
\hline $13: 25$ & 96.5 & 97 & Yellow Alert \\
\hline $13: 30$ & 97 & 97.5 & Yellow Alert \\
\hline $13: 35$ & 97.5 & 97.9 & Yellow Alert \\
\hline $13: 40$ & 97.9 & 98 & Yellow Alert \\
\hline $13: 45$ & 98 & 98.6 & Yellow Alert \\
\hline $13: 50$ & 98.6 & 99 & Yellow Alert \\
\hline $13: 55$ & 99 & 100 & Yellow Alert \\
\hline $14: 00$ & 100.1 & 100.3 & Yellow Alert \\
\hline $14: 05$ & 100.3 & 100.7 & Yellow Alert \\
\hline $14: 10$ & 100.7 & 101.1 & Yellow Alert \\
\hline $14: 15$ & 101.1 & 101.4 & Yellow Alert \\
\hline $14: 20$ & 101.4 & 102 & Yellow Alert \\
\hline $14: 25$ & 102 & 102.3 & Yellow Alert \\
\hline $14: 30$ & 102.3 & 102.9 & Yellow Alert \\
\hline $14: 35$ & 102.9 & 103.1 & Yellow Alert \\
\hline $14: 40$ & 103.1 & 103 & Yellow Alert \\
\hline $14: 45$ & 103 & 103 & Yellow Alert \\
\hline $14: 50$ & 103 & 102.8 & Yellow Alert \\
\hline $14: 55$ & 102.8 & 102.3 & Yellow Alert \\
\hline
\end{tabular}

\subsection{Chart}

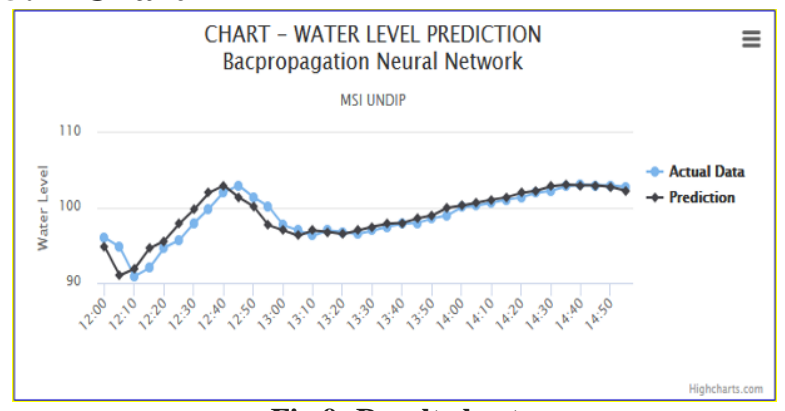

Fig 9: Result chart

The results of the water level prediction calculations performed using back propagation neural networks are then described in graphical form, as shown in figure 9. The graph used is a line graph. This line graph contains actual data lines, predictive data lines and fixed time ranges. This graph shows the actual data comparison with predicted results within the timeframe that has been set.

\section{CONCLUSION}

Based on the research that has been done, to produce some conclusions, among others are as follows: The results of the application of artificial neural network back propagation network algorithm for prediction of water level can produce accurate prediction, based on it, then the system designed can be used as a reference to make predictions the height of the water associated with the occurrence of floods. Implementation of back propagation neural network algorithm to predict water level produce MSE at the first iteration of 0.155569715812 , the smallest MSE that meets the treshold limit of 0.000099841 and $99.97 \%$ accuracy of data. This means that generally, the back propagation neural networks application produce accurate water level predictions, that is close to the actual data. 
The results of this study can be developed into an early warning system with the latest information technology using a smartphone technology that makes it easier to access disaster information before it happens.

\section{REFERENCES}

[1] Directorate General of Water Resources, 2016, Guidelines for the operation and maintenance of river Infrastructure and river maintenance, Circular letter No. 05/SE/D/2016 Ministry of Public of Works and Housing.

[2] Freeman, J. A., dan Skapura, D. M., 1992, Neural networks algorithms, applications, and programming techniques. Addison Wesley.

[3] Feng, L. H., Lu, J., 2010, The practical research on flood forecasting based on artificial neural networks. Expert Systems with Applications, 37, 2974-2977.

[4] Gallien, T.W., Schubert, J.E., Sanders, B.F., 2010, Predicting tidal flooding of urbanized embayments: A modeling framework and data requirements. Coastal Engineering 58 (2011) 567-577

[5] Kenyon, W., Hill, G., Shannon, P., 2008, Scoping the role of agriculture in sustainable flood management. Land Use Policy, 25, 351-360.

[6] Kusumadewi, S., 2004, Building Artificial Neural Networks Using Matlab and excelink. Jogjakarta. Graha Ilmu.

[7] Leea, T. L., Tsaib, C. P., Jengc, D. S., Shiehb, R. J., 2002, Neural network for the prediction and supplement of tidal record in Taichung Harbor, Taiwan. Advances in Engineering Software, 33, 329-338.
[8] Lyon, G. R., Fletcher, J. M., 2001, Early warning system. Education Matters, 1(2), 2-29.

[9] Meesuk, V., Vojinovic, Z., dan Mynett, A.E., 2012, Using Multidimensional Views of Photographs for Flood Modelling. Hydro and Agro Informatics Institute, Ministry of Science and Technology, 978-1-4673- 19751/12/\$31.00 @2012 IEEE.

[10] Mikhailov, V. N., Morozov, V. N., Cheroy, N. I., \& Mikhailova, M. V., 2008, Extreme flood on the Danube River in 2006. Russian Meteorology and Hydrology, 33, $48-54$.

[11] Rong-Kwei, L., dan Tzu-Chiang, L, 2005, A new ARTcounterpropagation neural network for solving a forecasting problem. Expert Systems with Applications.

[12] Suryono, Surarso, B., Saputra, R., 2014. Designing the Ultrasonic Ranger Telemetry System with the Wireless Sensor Network Model for the Flood and Rob Pattern Study. Semarang: UNDIP.

[13] Vivian F., López, Santo L., Medina, 2012, Taranis: Neural networks and intelligent agents in the early warning against floods, Expert Systems with Applications. 39, 10031-10037.

[14] Wiwin, S., dan Endang, S., 2009, Weather Pattern Generation for Flood Warning System, LIPI, Jakarta.

[15] Zheng, W., 2012, The Flood Monitoring Information System Framework Based on Multi-source Satellite Remote Sensing Data. 2012 International Conference on System Science and Engineering June 30-July 2, 2012, Dalian, China. 